\title{
Spontaneous patterning of quantum dots at the air-water interface
}

\author{
Richard P. Sear, Sung-Wook Chung, Gil Markovich, William M. Gelbart,* and James R. Heath ${ }^{\dagger}$ \\ Department of Chemistry and Biochemistry, University of California at Los Angeles, \\ 405 Hilgard Avenue, Los Angeles, California 90095-1569 \\ (Received 25 June 1998; revised manuscript received 8 March 1999)
}

\begin{abstract}
Nanoparticles deposited at the air-water interface are observed to form circular domains at low density and stripes at higher density. We interpret these patterns as equilibrium phenomena produced by a competition between an attraction and a longer-ranged repulsion. Computer simulations of a generic pair potential with attractive and repulsive parts of this kind, reproduce both the circular and stripe patterns. Such patterns have a potential use in nanoelectronic applications. [S1063-651X(99)50306-1]

PACS number(s): $68.10 .-\mathrm{m}$
\end{abstract}

Spontaneous formations of spatial patterns arise spectacularly and yet commonly in a wide variety of nonlinear kinetic [1] and biological [2] processes. Still, more interestingly, they are observed in a surprisingly diverse set of equilibrium situations involving fluids and solids in two and three dimensions. For example, in two dimensions (2D), systems as apparently disparate as magnetic garnets, Langmuir monolayers, and thin films of adsorbates all form periodic structures consisting of uniformly spaced domains with circular or striped shapes [3]. In the garnets, the magnetization normal to the plane is high inside these domains and low in between them whereas, in the Langmuir films, it is the number density of molecules that is different inside and outside the circles and stripes. The order parameter in these two cases is clearly different, but the spatial modulation - the pattern is the same. The size of the patterns, i.e., the period of their spatial modulation, varies from nm's (adsorbates on metals [4]) to micrometers (Langmuir films [5]) to cm's (ferrofluids [6]). In all of these cases, the onset of modulation, and its characteristic length scale, is caused by the competition between an attractive interaction and a longer-ranged repulsion; the former favors a bulk phase separation, while the latter transmutes this into a microphase transition, involving an equilibrated distribution of domains (e.g., circles, and stripes in 2D, and spheres, cylinders and lamellae in 3D $[3,7]$ ), all of which are of finite size in at least one direction.

In Langmuir monolayers, for example, the attractions are associated with van der Waals interactions between the alkyl chains (hydrophobic "tails") of the amphiphilic molecules at the surface of water. The longer-ranged repulsions arise from the oriented dipoles that comprise the hydrophilic "heads" of these species. This two-dimensional system has been modeled as a dipolar lattice gas by Hurley and Singer [8], and treated in the continuum limit by McConnell [9]. In many other instances of domain formation and microphase separation, the physical origin of the competing interactions is more subtle. In the case of diblock copolymers, for example, the effective repulsions are due to the fact that the two blocks are connected to each other, and it is this constraint that limits the size of the (spherical, cylindrical, and

\footnotetext{
*Electronic address: gelbart@ chem.ucla.edu

†Electronic address: heath@chem.ucla.edu
}

lamellar) domains [10]. In aqueous solutions of surfactant, the spontaneous appearance of micelles can be understood similarly in terms of the demixing between heads and tails being frustrated by their connectivity $[11,12]$. More explicitly, several groups $[13,14]$ have shown how the basic physics of structural organization and phase behavior in amphiphilic systems can be recast into lattice models involving ferromagnetic (attractive) nearest-neighbor interactions and antiferromagnetic (repulsive) next-nearest-neighbor interactions.

In this Rapid Communication we demonstrate that quantum dots - nanoparticles — at the air-water interface also organize spontaneously to form spatially modulated phases. We observe in particular that nanoparticles at submonolayer coverage form both circular and stripe domain patterns, depending on the extent of coverage. These structures are argued to be equilibrium states of the system, driven by the competition between attractions and longer-ranged repulsions between the nanoparticles. Computer simulation results confirm that a simple interaction potential consisting of these competing parts does indeed lead to the experimentally observed structures. While our results are related in fundamental ways to the spatial patterns reported for the many other systems mentioned above, we shall feature the concentration dependence of the observed spatial patterns. In particular, the reversible conversion from circles to stripes in $2 \mathrm{D}$, upon increase in nanoparticle coverage, is argued to be analogous to more general phenomena observed, for example, in surfactant solutions where micellar aggregates evolve from globular to cylindrical to bilayer forms as the volume fraction is raised [7]. In all of these cases, the interactions between the clusters are the ones that lead to their reorganization into lower-curvature shapes that can pack more efficiently.

In recent years, chemically synthesized quantum dots have begun to appear as the active elements in various prototype photonic $[15,16]$ and electronic $[17,18]$ devices. Simultaneous with these developments, techniques for the patterned assembly of nanoparticles via templating approaches have been reported [19-21]. Nontemplated, thermodynamically-controlled patterning can potentially produce organization at nanometer length scales that cover large areas. Such approaches may prove useful for certain defecttolerant device-related applications [22]. From a more fundamental point of view, the spherical symmetry of these par- 

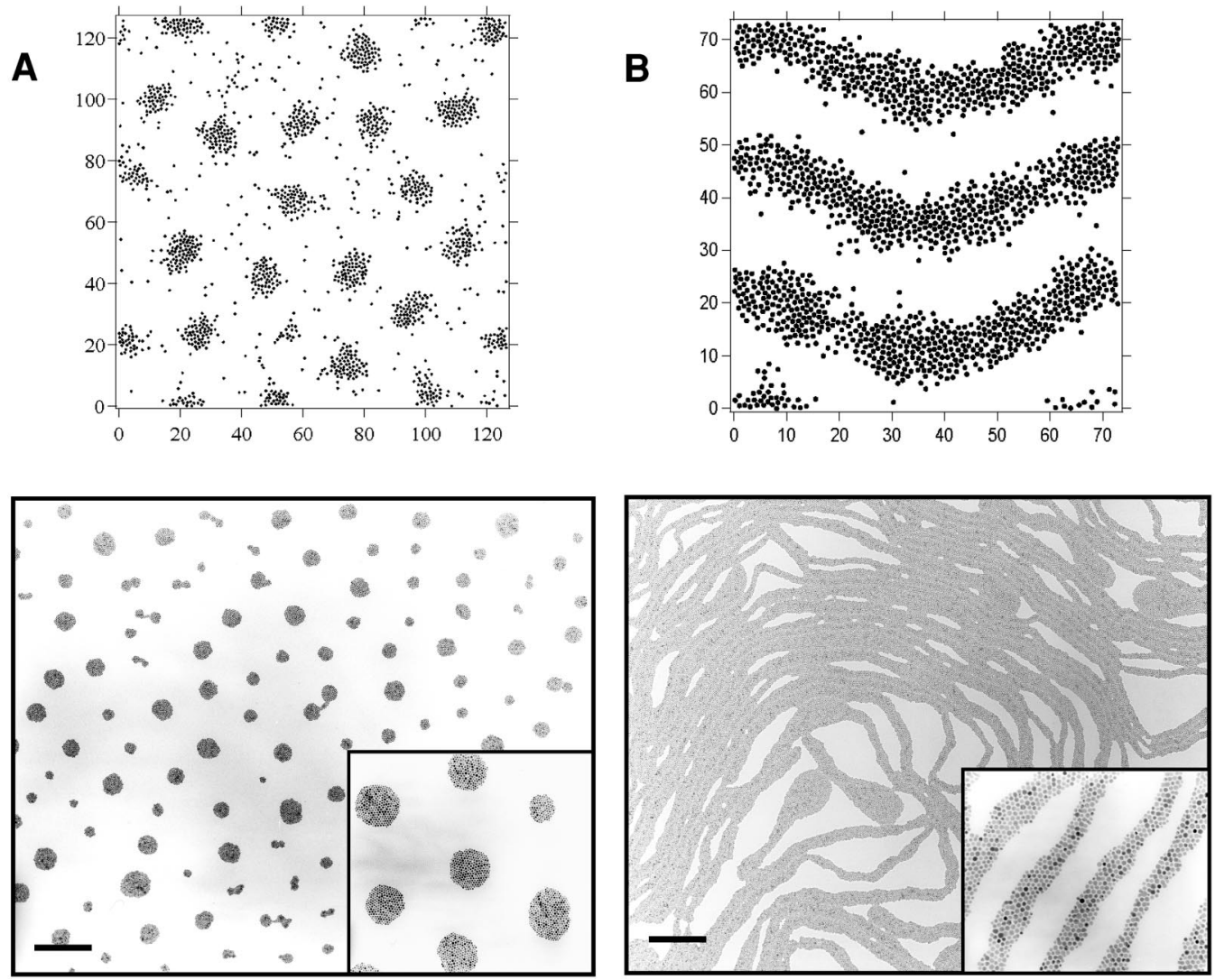

FIG. 1. Results of the computer simulation (top) for the potential of Eq. (1) and Fig. 2, at densities (a) $\rho=0.1$ and (b) $\rho=0.3$, and the corresponding TEM micrographs (bottom) revealing the spontaneous formation of clusters and stripelike arrays of alkylthiol passivated Ag nanocrystals. The solution used to prepare (b) was approximately three times more concentrated $(\approx 1 \mathrm{mg} / \mathrm{ml})$ than that of $(\mathrm{a})$. The size of the scale bar shown is $0.5 \mu \mathrm{m}$. The parameters of the potential are $\gamma_{a}=0.5, \gamma_{r}=0.25$, and $\epsilon_{r} / k T=\epsilon_{a} / k T=7$. The particles are represented by circles a little smaller than the diameter of the hard core, and the $x$ and $y$ axes show distances from the lower left corner of the simulation box, plotted in units of the hard-core diameter.

ticles makes modeling of their interactions and phase behavior easier than with complex molecular structures.

Circles and stripes of silver quantum dots were prepared using two different approaches. All experiments utilized hexane or heptane solutions of 4-6-nm-diameter Ag particles that were surface passivated with octanethiol. The structures of the (sub)monolayer films were sampled by transferring the Langmuir films to a transmission electron microscopy (TEM) grid using the horizontal lift-off (LangmuirSchaeffer) technique. In the first approach, several drops of a dilute $(0.3 \mathrm{mg} / \mathrm{ml})$ particle/hexane solution were evaporated on the water surface and the film was sampled at various stages of compression while simultaneously monitoring the pressure $(\pi)$ /area isotherm. Prior to monolayer compression (surface coverage $\approx 20 \%, \pi \approx 0 \mathrm{nN} / \mathrm{m}$ ) the particles were found to aggregate into circular domains [see the bottom of Fig. 1(a)], while at high surface coverage $(\approx 50 \%, \pi$ $\approx 1.5 \mathrm{nN} / \mathrm{m}$ ), a transition from circular domains to stripes [see the botton of Fig. 1(b)] has taken place. In the second type of experiment, a single drop of particle/hexane solution was deposited onto a clean water surface. Successive experiments were carried out by increasing the particle concentration in the drop from $\approx 0.1$ to $1 \mathrm{mg} / \mathrm{ml}$. When a single drop of particle solution is evaporated on the water surface, the coverage is highly non uniform. Macroscopic regions of particles, easily visible to the naked eye, are observed to form around the perimeter of the Langmuir trough. These regions were sampled as described above. As the particle concentration within the drops is increased from 0.3 to $0.5 \mathrm{mg} / \mathrm{ml}$, we observe a transition from circular domains to stripes. The fact that both circular-domain and the stripe structures were prepared using two distinct approaches, including one in which the islands were converted directly into stripes by compression [23], strongly suggests that these spatially modulated nanocrystal phases are at least metastable and can be described by an equilibrium model. As far as we are aware, this is the first demonstration of pattern formation in a system of spherically symmetric particles having a characteristic nanometer length scale [24], in which the patterns have been shown to be an equilibrium phenomenon.

In order to interpret the experimental results we assume that the particles interact via a pair potential $u(r)$, which is a function only of distance between the particles $r$. We chose a simple phenomenological form, plotted in Fig. 2:

$$
u(r)=u_{h c}(r)+u_{l r}(r),
$$

where $u_{h c}$ is a hard-core potential, equal to $\infty$ for $r<1$, the diameter of the hard core (taken as our unit of length), and 0 otherwise. $u_{l r}$ is the long-range part of the interaction, given by

$$
u_{l r}(r)=-\epsilon_{a} \gamma_{a}^{2} \exp \left(-\gamma_{a} r\right)+\epsilon_{r} \gamma_{r}^{2} \exp \left(-\gamma_{r} r\right)
$$




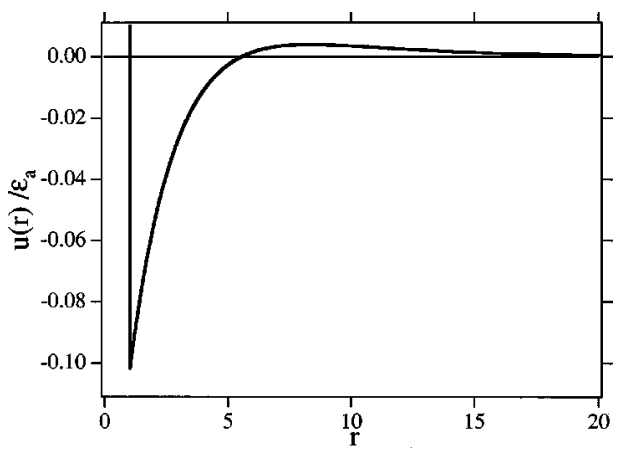

FIG. 2. The potential $u(r)$ [Eq. (1)], as a function of interparticle separation $r$, is shown by plotting it divided by $\epsilon_{a}$. The parameters of the potential are $\gamma_{a}=0.5, \gamma_{r}=0.25$, and $\epsilon_{r} / \epsilon_{a}=1$.

The first and second terms are, respectively, attractive and repulsive, $\epsilon_{a}, \epsilon_{r}>0$. The repulsive term is longer ranged than the attraction, $\gamma_{r}^{-1}>\gamma_{a}^{-1}$. Our motivation for choosing the hard-core part of the potential is obvious since the nanoparticles cannot interpenetrate one another. They also interact via a dispersion attraction, which we model with our attractive term of the Kac form [25]. The origin of the repulsion is much less obvious. As in other systems at the airwater interface $[5,24]$, it may be a dipole-dipole repulsion. The repulsion between the total dipole moments of the patches of water molecules beneath each nanoparticle is of the same order as the interaction strengths we use in the computer simulations [26]. This supports the idea of dipoledipole forces as the origin of the long-ranged repulsion, but does not, of course, prove it. In any case, the repulsion is taken here to be of the Kac form, to emphasize that its long range is a generic feature of systems that form equilibrium patterns; we shall further stress the generic nature of the observed progression from circular clusters to stripes upon an increase in particle concentration.

The computer simulations were performed using the standard Metropolis Monte Carlo algorithm [27] at a fixed number of particles, area, and temperature, with periodic boundary conditions. The number density $\rho=N / A$, with $N$ as the number of particles, is equal to 1600 in our simulations, and $A$ is the area in units of the square of the hard-core diameter. Due to the large length scale of the patterns and the small difference in free energy between different patterns, the simulations converged to equilibrium very slowly. We checked that our patterns were equilibrium structures by observing that our simulations converged to them starting from both a square lattice covering the whole area of the simulation box, and from a single large drop containing almost all of the particles at a liquidlike density. The results, presented in Fig. 1, are configurations obtained after 230 [Fig. 1(a)] and 440 [Fig. 1(b)] million Monte Carlo moves. It is easy to see that, at low density, both experiment and simulation [Fig. 1(a)], find (roughly) circular domains, while at high density [Fig. 1(b)], there are stripes. Qualitatively, we have complete agreement between experiment and simulation of the potential of Eq. (1).

Perhaps the simplest and most direct way to understand the domains and stripes is to consider them as disks or stripes of liquid whose small size is enforced by the long-ranged repulsion. As is well known, the attractive part of the poten- tial favors a vapor-liquid transition. This transition is driven by the reduction in energy at high densities where many particles are close enough to each other to feel their mutual attraction. However, with the long-ranged repulsion, the energy is only low for small clusters or stripes. When a cluster grows larger than about five diameters, the particles on opposite sides of the cluster start to repel each other, thereby increasing the energy.

We can make this idea more definite by considering the energy $u_{l i q}$ of one of our particles in a liquid, using the approximation of van der Waals [25]:

$$
u_{l i q}=\frac{1}{2} \rho \int u_{l r}(r) d \mathbf{r}=-\rho \pi\left(\frac{\epsilon_{a}}{k T}-\frac{\epsilon_{r}}{k T}\right),
$$

where $\mathbf{r}$ is a pair separation vector in $2 \mathrm{D}$. The computer simulations are for a potential with $\epsilon_{a}=\epsilon_{r}$, so within the van der Waals approximation [Eq. (3)], the energy is zero; the long-range attraction and repulsion cancel each other out for a bulk phase. Of course, Eq. (3) is only an approximation; nevertheless, while the energy of a dense fluid will be less than zero, it will not be much less than zero, and so we would not expect to see a liquid phase. This is consistent with the results of computer simulations in which no liquid phase has been seen.

In contrast, for widely separated circular domains of diameter $\lesssim 5$ particle diameters, no two particles are far enough apart to interact via the repulsive hump in the potential. Therefore, the attractive interactions will not be canceled out by the repulsion and the energy will be low. The energy is low because the density of particles is nonuniform.

At low density we do indeed find circular domains in both experiment and simulation. These domains are a little larger than five particle diameters, as their size is set by competition between the repulsion and an effective surface tension from the attractive interactions, which latter favors larger domains $[3,28]$. As the density increases, the domains are pushed together until there is a transition from domains to stripes. Roughly speaking, the domains can be pushed together until the interdomain separation is so small that each domain strongly repels the surrounding domains. The energy is then lower for stripes since, at any density, the separation between stripes is larger than that between circular domains.

In conclusion, nanoparticles deposited at the air-water interface form circular domains and stripes, depending on the density. The patterns have been described as resulting from competition between an attraction, which makes the particles aggregate, and a longer-ranged repulsion, which limits the aggregation to finite domains. The patterns are not new, insofar as many diverse systems form them [3], and the nanoparticles have much in common with these other systems. However, the fact that they are spherical particles and not, for example, molecules with a relatively complex architecture, makes them an unusually simple system to demonstrate such complex behavior. Also, we have emphasized the fact that an increase in concentration leads to a spontaneous reorganization of the self-assembled domains from circular clusters to stripes, as the repulsions between the aggregates become more important than those between the individual particles within them. This phenomenon is closely related to the transitions to hexagonal and lamellar phases observed 
commonly in concentrated surfactant solutions, where the locally preferred curvature of micelles is successively "squeezed out" of the systems as interaggregate repuslsions become dominant and the lower-curvature cylinder and bilayer geometries are found to better minimize the overall interaction free energy [7]. Finally, the patterns (as they are easily transferable to a variety of substrates), are not just important for understanding interactions at the air-water interface. For example, the stripe phase has been used as a shadow mask for electron beam lithography after an increase in the height of the stripe pattern to several particles [29]. The ability to control the conductivity of the particle monolayers [30,31], combined with the spontaneous formation of nanoscale "wires" from these particles [32], opens a signifi- cant pathway for the chemical assembly of nanoelectronic circuits. In the future, a better understanding of the nature of the competing interactions between nanoparticles at the airwater interface will allow us to optimize the size, conductivity, and alignment of the patterns, and to thus advance along that pathway.

We would like to acknowledge Sven E. Henrichs for preparing some of the Ag nanoparticles. This work was supported by the U.S. Office of Naval Research (ONR) under Order No. N00014-98-1-0422, and the American Chemical Society Petroleum Research Foundation (PRF) under Grant No. 31797-AC9. J.R.H. acknowledges support from the Packard Foundation and the Sloan Foundation.
[1] J.S. Langer, Rev. Mod. Phys. 52, 1 (1980); J. Ross and A.P. Arkin, J. Phys. Chem. 99, 10417 (1995).

[2] M. Falcke and H. Levine, Phys. Rev. Lett. 80, 3875 (1998).

[3] M. Seul and D. Andelman, Science 267, 476 (1995).

[4] P. Zeppenfeld, M. Krzyzowski, C. Romainczyk, G. Comsa, and M.G. Lagally, Phys. Rev. Lett. 72, 2737 (1994).

[5] D. Andelman, F. Brochard, C. Knobler, and F. Rondelez, in Micelles, Membranes, Microemulsions and Monolayers, edited by W.M. Gelbart, A. Ben-Shaul, and D. Roux (SpringerVerlag, New York, 1994).

[6] A.J. Dickstein, S. Erramilli, R.E. Goldstein, D.P. Jackson, and S.A. Langer, Science 261, 1012 (1993).

[7] A. Ben-Shaul and W.M. Gelbart, in Micelles, Membranes, Microemulsions and Monolayers (Ref. [5]).

[8] M.M. Hurley and S.J. Singer, Phys. Rev. B 46, 5783 (1992); J. Phys. Chem. 96, 1938 (1992); 96, 1951 (1992).

[9] H.M. McConnnell and R. Dekoker, Langmuir 12, 4897 (1996).

[10] F.S. Bates and G.H. Frederickson, Annu. Rev. Phys. Chem. 41, 525 (1990).

[11] F.H. Stillinger, J. Chem. Phys. 78, 4654 (1983).

[12] H.-J. Woo, C. Carraro, and D. Chandler, Phys. Rev. E 52, 6497 (1995).

[13] B. Widom, J. Chem. Phys. 84, 6943 (1986); K.A. Dawson, M.D. Lipkin, and B. Widom, ibid.. 88, 5149 (1988).

[14] G. Gompper and M. Schick, Self-Assembling Amphiphilic Systems, Vol. 16 in Phase Transitions and Critical Phenomena, edited by C. Domb and J.L. Lebowitz (Academic Press, London, 1994).

[15] N.C. Greenham, X. Peng, and A.P. Alivisatos, Phys. Rev. B 54, 17628 (1996).

[16] B.O. Dabbousi, M.G. Bawendi, O. Onitsuka, and M.F. Rubner, Appl. Phys. Lett. 66, 1316 (1995).

[17] G. Markovich, D.V. Leff, S.W. Chung, H.M. Soyez, B. Dunn, and J.R. Heath, Appl. Phys. Lett. 70, 3107 (1997).

[18] D.L. Klein, R. Roth, A.K.L. Lim, A.P. Alivisatos, and P.L. McEuen, Nature (London) 389, 699 (1997).

[19] R.W. Zehner, T.L. Morkved, H. Jaeger, and L.R. Sita, Langmuir 14, 242 (1998).
[20] P.C. Hidber, W. Helbig, E. Kim, and G.M. Whitesides, Langmuir 12, 1375 (1996).

[21] T. Vossmeyer, E. DeIonno, and J.R. Heath, Angew. Chem. Int. Ed. Engl. 36, 1080 (1997).

[22] J.R. Heath, P.J. Kuekes, G. Snider, and R.S. Williams, Science 280, 1717 (1998).

[23] The stripes shown in Fig. 1(b) were prepared by a compression of the lower concentration system [see Fig. 1(a)], resulting in some buckling of the stripes, as well as close contacts between them, due to the buildup of stress. The stripes prepared directly from a droplet of the higher concentration solution are found to be straighter and much more nearly uniformly spaced from one another [32].

[24] Similar behavior has been observed for micrometer-sized particles (polystyrene spheres) on the surface of water: J. RuizGarcia, R. Gamez-Corrales, and B. Ivlev, Physica A 236, 97 (1997); J. Ruiz-Garcia (private communication); and F. Ghezzi and J.C. Earnshaw, J. Phys.: Condens. Matter 9, L517 (1997).

[25] J.P. Hansen and I.R. McDonald, Theory of Simple Liquids, 2nd ed. (Academic Press, London, 1986).

[26] We estimate the repulsions as being between two parallel point dipoles interacting through water, each with a dipole moment 1000 times that of a water molecule; there are roughly 1000 water molecules underneath each nanoparticle. At distances of about ten particle diameters (see Fig. 2), this gives rise to a repulsive energy of about $0.01 \mathrm{kT}$.

[27] M.P. Allen and D.J. Tildesley, Computer Simulations of Liquids (Clarendon Press, Oxford, 1987).

[28] R.P. Sear and W.M. Gelbart, J. Chem. Phys. 110, 4582 (1999).

[29] S.H. Choi, M.S. Leung, G.W. Stupian, N. Presser, S.-W. Chung, G. Markovich, J.R. Heath and K.L. Wang (to be published).

[30] R.P. Andres, J.D. Bielefeld, J.I. Henderson, D.B. Janes, V.R. Kolagunta, C.P. Kubiak, W.J. Mahoney, and R.G. Osifchin, Science 273, 1690 (1996).

[31] G. Markovich, C.P. Collier, and J.R. Heath, Phys. Rev. Lett. 80, 3807 (1998).

[32] S.-W. Chung, G. Markovich, and J.R. Heath, J. Phys. Chem. B 102, 6685 (1998). 